\title{
Wigner-like crystallization of Anderson-localized electron systems with low electron densities
}

\author{
A. A. Slutskin ${ }^{1}$, M. Pepper ${ }^{2}$, and H. A. Kovtun ${ }^{1}$ \\ ${ }^{1}$ B. Verkin Institute for Low Temperature Physics and Engineering \\ of the National Academy of Sciences of Ukraine, 47 Lenin Ave., Kharkov 61103, Ukraine \\ E-mail: slutskin@theor.kharkov.ua \\ ${ }^{2}$ Cavendish Laboratory, University of Cambridge, Madingley Road, Cambridge CB3 OHE, UK
}

Received July 2, 2002

\begin{abstract}
We consider an electron system under conditions of strong Anderson localization, taking into account interelectron long-range Coulomb repulsion. We establish that at sufficiently low electron densities and sufficiently low temperatures the Coulomb electron interaction brings about ordering of the Anderson-localized electrons into a structure that is close to an ideal (Wigner) crystal lattice, provided the dimension of the system is $>1$. This Anderson-Wigner glass (AWG) is a new macroscopic electron state that, on the one hand, is beyond the conventional Fermi glass concept, and on the other hand, qualitatively differs from the known «plain» Wigner glass (inherent in self-localized electron systems) in that the random slight electron displacements from the ideal crystal sites essentially depend on the electron density. With increasing electron density the AWG is found to turn into the plain Wigner glass or Fermi glass, depending on the width of the random spread of the electron levels. It is shown that the residual disorder of the AWG is characterized by a multi-valley ground-state degeneracy akin to that in a spin glass. Some general features of the AWG are discussed, and a new conduction mechanism of a creep type is predicted.
\end{abstract}

PACS: 71.45.-d, 72.15.Rn, 73.20.-r, 73.40.-c

\section{Introduction}

As was shown by Wigner long ago [1], the slow decrease in the Coulomb electron-electron interaction potential $e^{2} / \kappa r$ ( $e$ is the free electron charge, $r$ is the distance between the interacting electrons, and $\kappa$ is the permittivity) with increasing interelectron distance inevitably causes the Coulomb energy of a free-electron gas to exceed its kinetic energy at sufficiently low electron densities, with the resulting transition of the gas into an electron crystal (Wigner crystal). In the wake of Wigner's prediction a natural question was raised whether long-range (weakly screened) Coulomb forces can lead to ordering of charge carrier ensembles in conductors. The experimental evidence that has been collected to date shows that it is very difficult (if possible at all) to observe the Wigner crystallization in the convential conductors. Therefore, charge carrier Coulomb ordering should be expected to exist first of all under conditions that the kinetic energies of the charge carrier movement over a conductor are sufficiently small.

At present much attention is being given to electron/hole lattice systems with so small an overlap integral that tunneling of the charge carriers between the host-lattice sites is supressed by their mutual Coulomb repulsion. Under these conditions a Coulomb self-localization of the electrons / holes inevitably takes place, bringing about their ordering in the following two cases: 1) If the host lattice is regular, an incommensurate electron/hole structure is generally formed for any charge carrier density [2,3]. 2) If the host lattice is disordered, but the mean separation of its sites, $a_{0}$, is much less than that of the electrons/holes $\bar{r}$, the ground-state space structure of the lattice gas of the charge carriers, though disordered, is obviously close to the corresponding Wigner crystal lattice (WCL), since the WCL spacing $a_{W} \sim \bar{r}$, while the random charge 
carriers' displacements from the WCL sites are $\sim a_{0}$. Such a randomly but slightly disordered WCL is often called Wigner glass*.

The present paper aims to draw attention to a new type of Coulomb ordering that can occur in Anderson-localized electron systems. (For definiteness, we consider the localized charge carriers to be electrons.) The Anderson localization is considered to be «strong» in the sense that the external random potential localizing the electrons exceeds the mean energy of the Coulomb repulsion per electron $\varepsilon_{C} \sim e^{2} / \kappa \bar{r}$. Without essential loss of generality the external potential can be concieved as a random set of potential wells (centers of electron localization), the spread of their electron levels $\Delta$ (the disorder energy) being $\gg \varepsilon_{C}$. The smallness of $\varepsilon_{C}$ as compared with $\Delta$ is believed to be a good reason to describe an influence of the Coulomb electron-electron interaction on the Anderson-localized systems in terms of a Fermi glass [5], a random system of the electrons occupying all wells with energies less than or equal to the Fermi energy $\varepsilon_{F}$. Up to now, the Fermi-glass approximation, modified to allow for the existence of the so-called Coulomb gap (Efros-Shklovskii gap) [6] at the Fermi energy, has been the basic approach to the problem. We show futher that at sufficiently low electron densities, $n_{e}$, and sufficiently low temperatures, $T$, the mutual Coulomb repulsion of the electrons inevitably forces the Anderson-localized electron ensemble to be arranged close to the WCL, provided the dimension of the system $d>1$. This Coulomb-ordered Anderson-localized electron system, which can be called an Anderson-Wigner glass ( $A W G$ ), is beyond the scope of the Fermi-glass concept.

There are strong reasons to suggest that the random spread of the energy levels of the Anderson-localized electrons results in a qualitative difference of the AWG from the above «plain» Wigner glass inherent to the Coulomb self-localized electron systems. As will be seen from the following, in an AWG, unlike the plain Wigner glass, the electron displacements from the WCL sites depend essentially on $n_{e}$. This imparts to the AWG a number of new interesting features. In particular, it will be shown below that an increase in $n_{e}$ turns an AWG into a Fermi glass or a plain Wigner glass depending on the value of the parameter $\gamma=\Delta / \varepsilon_{0}$ $\left(\varepsilon_{0}=e^{2} / \kappa r_{0}, r_{0}\right.$ is the mean separation of the wells) governing the interplay between the random spread of the electron levels and the Coulomb interelectron repulsion.

The residual disorder in AWG is further described in terms of dipole glass, a random system of interacting dipoles that issue from the WCL sites. The dipole representation of the disorder offered here turns out to be helpful for revealing a multi-valley degeneracy of the AWG ground state, reminiscent of that in spin glasses.

\section{The basic assumptions}

The quantum numbers of the Anderson-localized electron states are the radius vectors $\mathbf{R}$ of the wells' centers. These states will be denoted by $|\mathbf{R}\rangle$. The electron energies $\varepsilon(\mathbf{R})$ in the states $|\mathbf{R}\rangle$ (we will call them excitation energies) are random values, while the structure of the $\mathbf{R}$ set is assumed to be arbitrary; in particular, it can be regular. The lowest of the excitation energies is taken to be zero. As we are dealing with $\varepsilon(\mathbf{R})<\Delta$, the density $v$ of the number of excitation energies on the interval $[\varepsilon, \varepsilon+d \varepsilon]$ can be written in the form

$$
v=g n_{0} / \Delta
$$

where $n_{0} \sim r_{0}^{-d}$ is the density of the wells, and the constant $g \sim 1$. For simplicity, in that follows we put $g=1$.

In the considered limiting case of low electron densities we can neglect perturbations produced in the eigenstates of the system by the electron-electron interaction with accuracy to additions of the order of

$$
\lambda=\gamma^{-1}\left(\frac{r_{0}}{\bar{r}}\right)^{2} \exp \left(-\frac{r_{0}}{r_{l}}\right)<<1,
$$

where $r_{l} \lesssim r_{0}$ is the localization radius. In this approximation, the eigenstates can be identified with

$$
|\mathcal{R}\rangle=\left|\mathbf{R}_{1}\right\rangle\left|\mathbf{R}_{2}\right\rangle \ldots\left|\mathbf{R}_{N}\right\rangle,
$$

where $\mathcal{R}=\mathbf{R}_{1}, \ldots, \mathbf{R}_{N}$ denotes the set of wells occupied by the electrons, and $N$ is the number of electrons. The complete set of eigenstates comprises all possible $\mathcal{R}$ with different $\mathbf{R}_{1} \ldots \mathbf{R}_{N}$. With the same accuracy, the eigenvalues $E(\mathcal{R})$ corresponding to the states $|\mathcal{R}\rangle$ take the form

$$
E(R)=E_{C}(\mathcal{R})+E_{\mathrm{exc}}(\mathcal{R}),
$$

where

* As far as we know, such a system was first considered in the paper [4]. The term «Wigner glass» appeared later. 


$$
E_{C}=\frac{e^{2}}{2 \kappa} \sum_{\substack{i, k=1 \\ i \neq k}}^{N}\left|\mathbf{R}_{i}-\mathbf{R}_{k}\right|^{-1}
$$

is the energy of the mutual electron repulsion and

$$
E_{\mathrm{exc}}(\mathcal{R})=\sum_{i=1}^{N} \varepsilon\left(\mathbf{R}_{i}\right)
$$

is the total excitation energy, i.e., the energy of the noninteracting electrons localized at the wells.

We aim to find the structure of the electron configuration $\mathcal{R}_{g}$ that minimizes $E(\mathcal{R})$.

\section{Electron ordering and Fermi-glass instability}

Let us first discuss how mutual electron repulsion affects the ground state of the Fermi glass in the limit $n_{e} \rightarrow 0$. As $\varepsilon_{F}<<\Delta$, from Eq. (1) we obtain

$$
n_{e}=n_{0} \varepsilon_{F} / \Delta,
$$

i.e., $\varepsilon_{F}$ decreases linearly with $n_{e}$. Since $\varepsilon_{C}$ is proportional to $n_{e}^{1 / d}$, we find that for $d>1$ the ratio $\varepsilon_{F} / \varepsilon_{C} \propto n_{e}^{(d-1) / d}$ tends to zero with a decrease in $n_{e}$. Hence, as $n_{e}$ goes to zero, the Fermi glass becomes unstable with respect to mutual Coulomb repulsion of the electrons, the energy $E_{C}$ (Eq. (5)) dominating the excitation energies $\varepsilon(\mathbf{R})$.

As is known, the absolute minimum of $E_{C}$ is realized by a WCL. Since the electrons of Fermi glass are randomly arranged, the Coulomb energy per electron of the Fermi glass exceeds that of the WCL, $\varepsilon_{W}$, significantly, i.e., by an amount $\sim \varepsilon_{C}$. This suggests that for sufficiently low $n_{e}$ the configuration $\mathcal{R}_{g}$ falls into a class of $\mathcal{R}$ that meet the following conditions: i) for each WCL site there is an electron located in a small vicinity of the site, ii) the upper bound $\varepsilon_{b}$ of the excitation energies $\varepsilon\left(\mathbf{R}_{1}\right), \ldots, \varepsilon\left(\mathbf{R}_{N}\right)$ satisfies the inequalities

$$
\varepsilon_{F}<<\varepsilon_{b}<<\varepsilon_{C} .
$$

The energy per electron $\varepsilon_{\mathcal{R}}=E(\mathcal{R}) / N$ of such $\mathcal{R}$ is near $\varepsilon_{W}$. It cannot be less than $\varepsilon_{W}$, as $\varepsilon_{\mathrm{exc}}=E_{\mathrm{exc}} / N$ is positive (more precisely, it is $\left.\geq \varepsilon_{F}\right)$.

Any configuration of the above class is bound to belong to the set of points $\mathbf{R}$ at which $\varepsilon(\mathbf{R}) \leq \varepsilon_{b}$. The density of these points equals $n_{0}\left(\varepsilon_{b} / \Delta\right)$. Correspondingly, their mean separation is

$$
\rho\left(\varepsilon_{b}\right) \sim r_{0}\left(\Delta / \varepsilon_{b}\right)^{1 / d} .
$$

It is much less than the WCL spacing $a_{W} \sim \bar{r}$ owing to the first of the inequalities (7). Therefore, for each WCL site $\mathbf{m}$ there are inevitably several $\mathbf{R}$ of the set such that $|\mathbf{R}-\mathbf{m}| \sim \rho$. Populating these «proximate» states $|\mathbf{R}\rangle$ (one electron per site) yields just the configurations of the class mentioned above. For such a configuration the energy $\varepsilon_{\mathcal{R}}$ is the sum

$$
\varepsilon_{\mathcal{R}}=\varepsilon_{W}+\delta \varepsilon
$$

where $\delta \varepsilon$ is expected to be a small correction to $\varepsilon_{W}$. It consists of two terms:

$$
\delta \varepsilon=a\left(\frac{\rho}{\bar{r}}\right)^{2} \varepsilon_{W}+b\left(\frac{r_{0}}{\rho}\right)^{d} \Delta .
$$

The first term is the deformation energy produced by electron displacements over distances $\sim \rho$ from the WCL sites. The second term is $\varepsilon_{\text {exc }}$ expressed in terms of $\rho$ in view of Eq. (8) and the fact that $\varepsilon_{\text {exc }} \sim \varepsilon_{b}$. The factors $a=a(\mathcal{R}), b=b(\mathcal{R})$ depend on the details of $\mathcal{R}$, but they are both $\sim 1$ for any of the configurations considered.

The ground-state correction, $\delta \varepsilon_{g}$, to $\varepsilon_{W}$ is the least of the $\delta \varepsilon$ values. Putting $a, b=1$ in the expression (10) and finding its minimum with respect to $\rho$, we obtain the estimate

$$
\delta \varepsilon_{g} \sim \gamma^{\frac{2}{d+2}}\left(\frac{r_{0}}{\bar{r}}\right)^{\frac{2(d-1)}{d+2}} \varepsilon_{W},
$$

the minimum being reached at

$$
\rho=\bar{\rho} \sim \gamma^{\frac{1}{d+2}}\left(\frac{r_{0}}{\bar{r}}\right)^{\frac{d-1}{d+2}} a_{W} \sim\left(\frac{\Delta}{\delta E_{C}}\right)^{\frac{1}{d+2}} r_{0},
$$

where $\gamma$ is the parameter defined in Sec. 1; $\delta E_{C}=\left(r_{0} / \bar{r}\right)^{2} \varepsilon_{C}$; the physical sense of this energy parameter will be explained below (Sec. 5). The quantity $\bar{\rho}$ characterizes the deviation of $\mathcal{R}_{g}$ from the WCL.

Expressions (11) and (12) show that $\delta \varepsilon_{g} / \varepsilon_{W}$ and $\bar{\rho} / a_{W}$ both go to zero when $\bar{r} / r_{0} \rightarrow \infty$. Hence, for sufficiently low $n_{e}$ the ground-state electron configuration is the WCL slightly perturbed by random electron displacements from the WCL sites. This is just the AWG mentioned in the Sec. 1. The space structure of the AWG differs from that of the plain Wigner glass in Coulomb self-localized electron systems (Sec. 1) in an important aspect: the typical displacement of the electrons/holes from the WCL sites in the plain Wigner glass is of the order of the geometrical constant $a_{0}$, while that in the AWG, $\bar{\rho}$ (Eq. (12)), depends not only on the geo- 
metrical parameter $r_{0}$ but also on both the disorder energy $\Delta$ and the electron density.

It should be noted that estimates (11) and (12) can be rewritten in the form

$$
\frac{\bar{\rho}}{a_{W}} \sim\left(\frac{\varepsilon_{F}}{\varepsilon_{C}}\right)^{\frac{1}{d+2}}, \quad \frac{\delta \varepsilon_{g}}{\varepsilon_{W}} \sim\left(\frac{\varepsilon_{F}}{\varepsilon_{C}}\right)^{\frac{2}{d+2}} .
$$

It follows herefrom that smallness $\varepsilon_{F}$ as compared with $\varepsilon_{C}$ is sufficient for the AWG to come into existence.

\section{Dipole glass}

To gain better insight into the «residual» disorder of the AWG ground state it is necessary to consider all electron configurations $\mathcal{R}$ that are close to $\mathcal{R}_{g}$. To this end, it is appropriate to map these electron configurations on the sets of dipoles issuing from the WCL sites. The mapping is based on the fact that each pair

\section{a neutral (empty) site of the WCL + the electron}

of a given configuration nearest to the site

is the same as the pair

$$
\text { the site with the electron }+ \text { dipole } e \mathbf{d}_{\mathbf{m}} \text {, }
$$

where the dipole vector $\mathbf{d}_{\mathbf{m}}=\mathbf{R}-\mathbf{m}, \mathbf{R}$ and $\mathbf{m}$ are the radius vectors of the electron and the site, respectively. In dipole terms the Hamiltonian (4) takes the form

$$
E(\mathcal{R})=N \varepsilon_{W}+H_{\text {eff }},
$$

where the effective Hamiltonian $H_{\text {eff }}$ is a functional of the dipoles $\mathbf{d}_{\mathbf{m}}$ as the independent variables. Taking into account that $\left|\mathbf{d}_{\mathbf{m}}\right|<<a_{W}$ for the configurations considered and expanding $E_{C}$ in powers of $d_{\mathrm{m}}^{\alpha}$ (the index $\alpha$ enumerates the components of vectors), in the quadratic approximation we obtain

$$
H_{\mathrm{eff}}=\sum_{\mathbf{m}} \tilde{\varepsilon}\left(\mathbf{d}_{\mathbf{m}}\right)+\frac{e^{2}}{2 \kappa} \sum_{\mathbf{m}, \mathbf{m}^{\prime}} \Lambda_{\alpha \alpha^{\prime}}\left(\mathbf{m}-\mathbf{m}^{\prime}\right) d_{\mathbf{m}}^{\alpha} d_{\mathbf{m}^{\prime}}^{\alpha^{\prime}}
$$

Here the matrix

$$
\Lambda_{\alpha \alpha^{\prime}}(\mathbf{m})=|\mathbf{m}|^{-3}\left(\delta_{\alpha \alpha^{\prime}}-3 m_{\alpha} m_{\alpha^{\prime}} /|\mathbf{m}|^{2}\right)
$$

determines the interaction of two dipoles issuing from sites $0, \mathbf{m}$; the function

$$
\tilde{\varepsilon}\left(\mathbf{d}_{\mathbf{m}}\right)=\varepsilon\left(\mathbf{m}+\mathbf{d}_{\mathbf{m}}\right)+\frac{e^{2}}{2 \kappa} \bar{\Lambda}_{\alpha \alpha^{\prime}} d_{\mathbf{m}}^{\alpha} d_{\mathbf{m}}^{\alpha^{\prime}},
$$

where

$$
\bar{\Lambda}_{\alpha \alpha^{\prime}}=-\sum_{\mathbf{m}} \Lambda_{\alpha \alpha^{\prime}}(\mathbf{m}),
$$

here and below, summation over repetitive indexes $\alpha, \alpha^{\prime}$ is implied, and the sums of $\mathbf{m}, \mathbf{m}^{\prime}$ are taken over all WCL sites. The second term in $\tilde{\varepsilon}\left(\mathbf{d}_{m}\right)$ is the energy of interaction of a given dipole with the WCL; the second term in Eq. (14) is the energy of the dipole-dipole interaction.

The Hamiltonian (14) has sense provided that $H_{\text {eff }} \ll N \varepsilon_{W}$. This is true if not only $\left|\mathbf{d}_{\mathbf{m}}\right|<<a_{W}$ but also $\varepsilon\left(\mathbf{m}+\mathbf{d}_{\mathbf{m}}\right)<<\varepsilon_{C}$. As follows from the previous Section, the dipoles $\mathbf{d}_{\mathbf{m}}$ meeting these requirements are bound to form some random set, i.e., the dipole system considered is in fact a dipole glass. There are good reasons to suggest that the dipole glass has much in common with the spin glass [7]. The similarity becomes clear if one considers a fairly simplified model in which $\mathbf{d}_{\mathbf{m}}=d_{0} \mathbf{e}_{\mathbf{m}} s_{\mathbf{m}}$, the independent variables $s_{\mathbf{m}}= \pm 1, d_{0}$ is a constant, and the unit vectors $\mathbf{e}_{\mathbf{m}}$ constitute some given random set. In such a case $H_{\text {eff }}$ takes the form

$$
H_{\mathrm{eff}}=\sum_{\mathbf{m} \neq \mathbf{m}^{\prime}} J_{\mathbf{m m}^{\prime}} s_{\mathbf{m}} s_{\mathbf{m}^{\prime}}+\sum_{\mathbf{m}} h\left(s_{\mathbf{m}}\right),
$$

where $s_{\mathrm{m}}$ play the role of «spins», $J_{\mathbf{m} \mathbf{m}^{\prime}}=d_{0}^{2} \Lambda_{\alpha \alpha^{\prime}}\left(\mathbf{m}-\mathbf{m}^{\prime}\right) e_{\mathbf{m}}^{\alpha} e_{\mathbf{m}^{\prime}}^{\alpha^{\prime}}$, a random function of $\mathbf{m}, \mathbf{m}^{\prime}$, is correspondingly an «exchange integral»; the function $h\left(s_{\mathbf{m}}\right)=\tilde{\varepsilon}\left(d_{0} \mathbf{e}_{\mathbf{m}} s_{\mathbf{m}}\right)$ can be considered as an external random field. The system with the Hamiltonian (15) falls into the class of spin glasses, possessing their known general property: a multi-valley ground-state degeneracy [7]. The real dipole Hamiltonian (14) differs from the model one (15) only in that each dipole $\mathbf{d}_{\mathbf{m}}$ meeting the above conditions runs through a given finite random set that comprises more than two vectors. Therefore, the multi-valley degeneracy of the dipole-glass ground state holds in the general case.

An adequate description of the above-mentioned

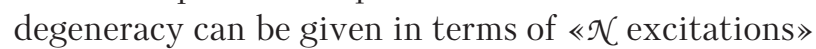
that are dipole configurations which contain $\mathcal{N}>1$ dipoles other than those in the ground state. The total number $Z$ of the $\mathcal{N}$ excitations is exponentially large in $\mathcal{N}$, while an increase in the dipole glass energy produced by an $\mathcal{N}$ excitation cannot exceed $\mathcal{N} \varepsilon_{C}$. Therefore, the typical separation between the neighboring energies of the $\mathcal{N}$-excitation energy spectrum, $\delta E(\mathfrak{N}) \propto \mathcal{N} / Z$, is exponentially small in $\mathcal{N}$. Due to the dipole-glass randomness the gap between the lower bound of the $\mathcal{N}$-excitation spectrum and the ground-state energy, $\delta E_{g}(\mathcal{N})$, is comparable with $\delta E(\mathcal{N})$. This just results in the multi-valley degeneracy of the dipole glass ground state, since in 
the limit $N \rightarrow \infty$ there are an infinite set of $\mathcal{N}$ excitations (《valleys») whose energies differ from that of the ground state only by exponentially small (in $N)$ values, their dipole arrangements having nothing in common with one another.

\section{The AWG existence region}

Estimates (12) show that ratio $\bar{\rho} / a_{W}$ is a monotonically increasing function of $n_{e}$, though $\bar{\rho}$ itself is a monotonically decreasing one. This brings about, depending on the value of the parameter $\gamma$, two different scenarios of what happens with the AWG as $n_{e}$ increases. If $\gamma>1$, the typical electron deviation from the WCL sites $\bar{\rho}$ inevitably reaches values $\sim a_{W}$ with an increase in $n_{e}$, the ratio $\varepsilon_{F} / \varepsilon_{C}$, as is seen from Eq. (13), becoming $\sim 1$. This allows the conclusion that there exists some critical $n_{e}=n_{c 1}$ at which the AWG turns into a Fermi glass (supposedly, by a second-order phase transition). Denoting by $\beta$ the critical ratio $\bar{\rho} / a_{W}$ corresponding to $n_{c 1}$ (it is bound to be $<1 / 2$ ), we find from Eq. (12)

$$
n_{c 1} \sim \beta^{\frac{d(d+2)}{d-1}} \gamma^{-\frac{d}{d-1}} n_{0} .
$$

For $\gamma<<1$, an increase in $n_{e}$ reduces $\bar{\rho}$ down to its least possible value, $r_{0}$, the ratio $\bar{\rho} / a_{W}$ remaining $<1$. This occurs for $\Delta \sim \delta E_{C}$. The parameter $\delta E_{C}$ is the typical change in the Coulomb energy of the system as an electron is shifted over a distance $\sim r_{0}$. Therefore, if $\delta E_{C} \gg \Delta$, the random spread of the electron levels can be neglected to good accuracy, and the arrangement of the Anderson-localized electrons is entirely determined by the mutual electron repulsion*. In such a case the space structure of the Anderson-localized electron system is in fact the same as that of the self-localized systems discussed in Sec. 1, i.e., it is an incommensurate electron structure [3] (the potential wells are regularly arranged) or plain Wigner crystal [4] (a disordered arrangement of the wells). Hence, for sufficiently small $\gamma$ the AWG is bound to turn into a structure of the self-localized type at some critical $n_{e}=n_{c 2}$ meeting the condition $\Delta \sim \delta E_{C}$. From the second of the estimates (12) we have

$$
n_{c 2} \sim \gamma^{d / 3} n_{0} .
$$

It follows from the aforesaid that the AWG existence region on the $n_{e}, \gamma$ plane is bounded by the $\gamma$ axis and the two curves $n_{e}=n_{c 1}(\gamma)$ and $n_{e}=n_{c 2}(\gamma)$ $\left(n_{c 1}(\gamma) \rightarrow 0\right.$ as $\left.\gamma \rightarrow \infty ; n_{c 2}(0)=0\right)$. They intersect at the point $n_{e}=\bar{n}_{e} \sim \beta^{d} n_{0}, \quad \gamma=\bar{\gamma} \sim \beta^{3}$, the value $\bar{n}_{e}$ being the maximal $n_{e}$ at which the AWG exists.

Heating affects the AWG if $T$ exceeds $\delta \varepsilon_{g}$, both terms in Eq. $(10)(\rho=\bar{\rho})$ being $\sim T$. Under these conditions the dipole thermal-fluctuations amplitude $d_{T} \sim \bar{r}\left(\varepsilon_{C} / T\right)^{1 / 2}$, the fluctuating dipole vector taking $\sim\left(T / \delta \varepsilon_{g}\right)^{1+d / 2}$ values. As $T$ increases, $d_{T}$ becomes $\sim a_{W}$, and at some critical $T \sim \varepsilon_{C}$ the AWG turns into a glassy state.

\section{Some general features of the AWG}

Macroscopically, the AWG manifests both electron crystal and glassy-state features.

Due to the proximity of the AWG space structure to a WCL the only possible one-electron excitations that can transfer charge in the AWG are point defects, positively charged the WCL vacancies, and interstitial electrons. In other words, the one-electron variable-range hopping that is inherent to the Fermi glass [5] is impossible in the AWG. In this respect the AWG is similar to an ideal Wigner crystal.

Conduction in the AWG is by two mechanisms: i) transfer of charged point defects, ii) multi-electron processes of a creep type that reside in the dipole glass. Here we only outline them.

The above point defects form an ideal gas whose conductivity $\sigma_{\text {def }}$, being proportional to the concentration of defects, depends on the inverse temperature exponentially to within a pre-exponential factor:

$$
\sigma_{\mathrm{def}} \propto \exp \left(\frac{\varepsilon_{v}-\varepsilon_{i}}{T}\right) .
$$

Here $\varepsilon_{v} \sim \varepsilon_{C}$ and $\varepsilon_{i} \sim \varepsilon_{C}$ are the energies of vacancy and interstitial-electron formation, respectively $\left(\varepsilon_{i}>\varepsilon_{v}\right)$. It is interesting that at sufficiently low temperatures the pre-exponential factor obeys the Mott's law for the Fermi glass [5]. Namely, $T$ is bound to be less than the quantity $\delta \varepsilon_{g}<<\varepsilon_{C}$ (Eq. (11)), which is the typical value of the random spread of the electron energies in an AWG. With respect to the hopping of a point defect bet-

* To avoid misunderstanding, it should be noted that the criterion of Anderson localization $t<\Delta$ ( $t$ is the overlap integral for wells separated by a distance $r_{0}$ ) is implicitly, assumed to be fulfilled irrespective of the relationship between $\delta E_{C}$ and $\Delta$. 
ween WCL sites $\delta \varepsilon_{g}$ plays the same role as the disorder energy of the familiar Mott's picture.

The conduction of the second type is by a creep in the dipole glass (Sec. 4) produced by the creation and annihilation of $\mathcal{N}$ excitations as phonons are adsorbed and emitted by AWG electrons. It is essential that only those $\mathcal{N}$ excitations take part in the charge transfer whose energies $E_{\mathcal{X}}$ are exponentially close in $\mathcal{N}$ to the AWG ground-state energy $E_{g}$. (According to Sec. 4 just these excitations cause the infinite degeneracy of the AWG ground state). It is just the exponetial smallness of $E_{\mathcal{N}}-E_{g}$ that makes possible the creation and annihilation of $\mathcal{N}$ excitations by one-phonon absorption and emission at temperatures as low as desiried. The corresponding $\mathcal{N}$ are determined by the relation $E_{\mathcal{X}}-E_{g} \sim T$. This gives $\mathcal{N} \sim \ln \left(\varepsilon_{C} / T\right)$. Taking into account that the quantum amplitude of transition (produced by the electron-phonon interaction) between the ground state and an $\mathcal{N}$ excitation is proportional to $\lambda^{\mathcal{N}}$ (the parameter $\lambda<<1$ has been defined in Sec. 2, Eq. (2)), we find the contribution $\sigma_{\text {creep }}$ made by the creep mechanism to the AWG conductivity to be given by the following estimate:

$$
\sigma_{\text {creep }} \propto\left(T / \varepsilon_{C}\right)^{-2|\ln \lambda|} .
$$

As $\sigma_{\text {creep }}$ depends on $T$ by a power law, it exceeds $\sigma_{\text {def }}$ at sufficiently low temperatures.

The multi-valley degeneracy of the dipole glass, similarly to that in a spin glass [7], is bound to cause an infinite spectrum of relaxation times, and, in consequence of this, nonergodic behavior of the AWG. This can be revealed by the observation of various relaxation processes in the AWG that go on for anomalously long times. An example is the relaxation of a nonequilibrium AWG polarization created in one way or another. This has much in common with relaxation of a nonequilibrium magnetic moment in spin glass.

As follows from the evaluations made in Sec. 5, under moderate-disorder conditions $(\gamma \lesssim 1)$ the
AWG can exist up to values of $n_{e}$ that are only several times less than $n_{0}$. The proper materials to observe the AWG are various amorphous narrow-band conductors, superlattices, and inversion layers wherein $n_{e}$ can be varied over wide limits without affecting the disorder. Favorable conditions for semiconductor AWGs are expected to exist in superlattices with the so-called $\delta$ layers. Of special interest is a conductive sheet in a metal-n-type GaAs$p$-type GaAs system with charge transfer in an impurity band [8]. It is distinguished by pronounced regular-type oscillations in $n_{e}$, which cannot be explained in conventional one-electron terms and most likely are a manifestation of a two-dimensional AWG. We intend to provide new evidence to confirm the suggestion and to specify the electron structure in the very nearest future.

The main outlines of the AWG mentioned here are to be the subjects of our further detailed publications.

We gratefully acknowledge interesting and useful discussions with I. Lerner and I. Yurkevich. We are sincerely thankful to B. Shklovskii for helpful remarks.

The visit of A. Slutskin to the Cavendish Laboratory was supported by the Royal Society. A. S. would like to express his gratitude.

1. E. Wigner, Trans. Farad. Soc. 34, 678 (1938).

2. J. Hubbard, Phys. Rev. B17, 494 (1978).

3. A. A. Slutskin, V. V. Slavin, and H. E. Kovtun, Phys. Rev. B61, 14184 (2000).

4. M. S. Bello, E. I. Levin, B. I. Shklovskii, and A. L. Efros, JETP 53, 822 (1981).

5. N. F. Mott and E. A. Davis, Electron Processes in Non-crystalline Materials, Clarendon Press, Oxford (1979).

6. A. L. Efros and B. I. Shklovskii, J. Phys. C8, L49 (1975).

7. K. Binder and A. P. Young, Rev. Mod. Phys. 58, 801 (1986).

8. M. Pepper, J. Phys. C12, L617 (1979). 